(2) Open Access Full Text Article

\title{
Lymphoma-targeted treatment using a folic acid- decorated vincristine-loaded drug delivery system
}

This article was published in the following Dove Press journal:

Drug Design, Development and Therapy

\author{
Lei Qiu' \\ Chao Dong ${ }^{2}$ \\ Xuan $\mathrm{Kan}^{3}$ \\ 'Department of Internal Medicine \\ Oncology, Shandong Cancer Hospital \\ Affiliated to Shandong University, \\ Shandong Academy of Medical Science, \\ Ji'nan, Shandong Province, People's \\ Republic of China; ${ }^{2}$ Department of \\ Oncology, 105 Hospital of People's \\ Liberation Army, Heifei, Anhui \\ Province, People's Republic of China; \\ ${ }^{3}$ Department of Oncology, Hospital \\ of Traditional Chinese Medicine of \\ Laiwu City, Laiwu, Shandong Province, \\ People's Republic of China
}

Purpose: B-cell lymphoma is the most frequently diagnosed lymphoid tumor. Folic acid (FA)-decorated systems were found to be preferentially internalized on the B-cell lymphoma cell line which is reported to express the folate receptor. This study was designed to develop an FA-decorated vincristine (VCR)-loaded system for targeted lymphoma treatment.

Methods: FA-decorated lipid was synthesized. VCR-loaded lipid-polymer hybrid nanoparticles (LPNs) were fabricated. In vitro cell lines and an in vivo lymphoma animal model was used to evaluate the anti B-cell lymphoma effect.

Results: FA-decorated, VCR-loaded LPNs (FA-VCR/LPNs) have shown a targeted effect in delivery to B-cell lymphoma cells. FA-VCR/LPNs also showed the highest anti-tumor effect in murine-bearing lymphoma xenografts.

Conclusion: FA-VCR/LPNs can achieve targeted delivery of VCR, bring about an outstanding therapeutic effect to treat lymphoma, and also reduce the systemic toxicity. FA-VCR/LPNs could be an excellent system for lymphoma therapy.

Keywords: lymphoma, drug delivery system, lipid-polymer hybrid nanoparticles, folic acid, vincristine

\section{Introduction}

Non-Hodgkin lymphoma (NHL) is a type of blood cancer, which accounts for over $85 \%$ of all lymphoma and approximately $4 \%$ of all malignancies. ${ }^{1-3}$ Although chemotherapy plays a vital role in treating NHL, the clinical applications are limited because of intolerable side-effects and multi-drug resistance. ${ }^{2}$ Therefore, nanotechnology has recently proved to be a promising therapeutic platform for lymphoma.

Compared to free drugs, incorporating drugs into nanocarriers can increase circulation time and reduce exposure to healthy tissue and, even more, enhance anti-tumor efficacy and reduce side-effects. ${ }^{4,5}$ Liposomes and nanoparticles have been widely applied for carrying free drugs for the treatment of lymphoma. DepoCyt ${ }^{\circledR}$ (Pacira Pharmaceuticals Inc., San Diego, CA, USA) (liposomal cytarabine) has been approved by the US Food and Drug Administration for the treatment of lymphoma. Numerous preclinical and clinical trials involving nanoparticles for the treatment of lymphoma are ongoing or have been recently completed: albumin-bound paclitaxel, liposomal vincristine, liposomal doxorubicin, and liposomal cytarabine and polyethylene glycol (PEG)-asparaginase in modified “Linker" Regimen; CD22-targeted PEGylated liposomes, multivalent rituximab lipid nanoparticles, PEGylated poly(lactic-co-glycolic acid) (PLGA) nanoparticles, etc. ${ }^{6-9}$

Lipid-polymer hybrid nanoparticles (LPNs) are core-shell nanoparticle structures comprising polymer cores and lipid/lipid-PEG shells, which exhibit complementary characteristics of both polymeric nanoparticles and liposomes, particularly in terms 
of their physical stability and biocompatibility. ${ }^{10}$ Based on their distinct functional structures, LPNs have exhibited some unique advantages: high structural integrity, improving drug stability during storage, controlled drug release, and high biocompatibility, while excluding some liposomes' or nanoparticles' limitations. ${ }^{11-13}$ In this study, LPNs were chosen as the carrier to deliver the hydrophobic drug vincristine (VCR).

Different targeting strategies are currently under investigation to enhance the specificity of anti-lymphoma drug carriers, including antibodies, antibody fragments, peptides, folic acid (FA), etc. ${ }^{14-17}$ FA has been used as a targeting device because of the folate receptor, which is highly overexpressed in various tumors and lower in normal tissue. Therefore, LPNs functionalized with FA are studied in this research.

VCR, widely used clinically to treat cancers including acute lymphoblastic leukemia, cervical and breast cancer, is a hydrophobic alkaloid derived from Catharanthus roseus. ${ }^{18}$ However, severe side effects such as dose-dependent neurotoxicity and multidrug resistance have limited its clinical application. To the best of our knowledge, there is no published study on folic acid-decorated LPNs drug delivery system for VCR delivery.

In this study, FA-decorated VCR LPNs were designed and researched. FA-decorated lipid was synthesized. VCRloaded LPNs were fabricated and applied to lymphoma in vitro on lymphoma cell lines and in vivo on a lymphoma animal model. Anti-cancer therapeutic effects and reduced systematic toxicity are expected for this system.

\section{Materials and methods Materials}

VCR, FA, stearic acid (SA), 3-(4,5-dimethylthiazol-2-yl)2,5-diphenyltetrazolium (MTT), dicyclohexylcarbodiimide (DCC), dimethylaminopyridine (DMAP), and dimethyl sulfoxide (DMSO) were provided by Sigma-Aldrich (St Louis, $\mathrm{MO}, \mathrm{USA}$ ). $\mathrm{NH}_{2}-\mathrm{PEG}_{5000}-\mathrm{COOH}$ was obtained from Beijing Kaizheng United Medical Technology Co., Ltd (Beijing, People's Republic of China). PLGA (50:50, molecular weight: 24,000-38,000) was purchased from Jinan Daigang Biomaterial Co., Ltd (Jinan, People's Republic of China).

\section{Cells}

Human Burkitt's lymphoma cell line (Raji cells), B-cell lymphoma cell line (A20 cell line), and human umbilical vein endothelial cells (HUVEC) were purchased from the American Type Culture Collection (ATCC, Manassas, VA, USA). Roswell Park Memorial Institute
(RPMI)-1640 medium was used for the culture of cells along with $1 \%(\mathrm{v} / \mathrm{v})$ of penicillin/streptomycin solution; $2 \mathrm{mg} / \mathrm{mL}$ of sodium bicarbonate; and $0.05 \mathrm{mM}$ of 2-mercaptoethanol. Exposing Raji cells to VCR solutions could provide the vincristine-resistant lymphoma cell line (Raji/VCR cells). ${ }^{19}$

\section{Animals}

All animal experiments were conducted according to protocols approved by Shandong University and complied with the National Institutes of Health Guide for the Care and Use of Laboratory Animals (NIH Publications No 8023, revised 1978). The in vivo experiments were approved by the Medical Ethics Committee of Shandong University (No 100120170076). BALB/c nude mice (4-6 weeks, 18-22 g) were provided by the Medical Animal Test Center of Shandong University (Ji'nan, People's Republic of China). Raji/VCR cells $\left(5 \times 10^{7}\right.$ cells in $100 \mu \mathrm{L}$ PBS $)$ were injected subcutaneously on the right flanks of each mouse. Tumor volumes were estimated using the following equation: $\left(\right.$ length $\times$ width $\left.^{2}\right) / 2$.

\section{Synthesis of FA-decorated lipid}

FA-decorated lipid was designed and synthesized using $\mathrm{NH}_{2}$ PEG-COOH as a linker to conjugate FA and SA (Figure 1). ${ }^{15}$ $\mathrm{SA}$ was dissolved in DMSO, then $\mathrm{NH}_{2}-\mathrm{PEG}-\mathrm{COOH}$ (1:1 with $\mathrm{SA}$, molar ratio), followed by adding DCC and DMAP into the DMSO solution in an ice bath. After stirring at $400 \mathrm{rpm}$ at room temperature for $6 \mathrm{~h}$, PEG-SA was formed. FA (1:1 with SA, molar ratio) dissolved in DMSO was finally added along with DCC and DMAP into the PEG-SA solution, and stirred at $400 \mathrm{rpm}$ for $6 \mathrm{~h}$ at room temperature to get FA-PEG-SA. The chemical structure was confirmed using nuclear magnetic resonance $\left({ }^{1} \mathrm{H}-\mathrm{NMR}\right)$ spectroscopy.

\section{Fabrication of LPNs}

FA-decorated LPNs (FA-LPNs) with polymeric PLGA cores and lipid shells were fabricated by the ultrasonication method (method A). ${ }^{20}$ The oil phase was prepared by dissolving PLGA in dichloromethane. The aqueous phase was prepared by dispersing FA-PEG-SA in ultrapure water by sonication. The oil phase was then poured into the water phase and the mixture was sonicated by probe ultrasonicator in a ice bath. The resulting suspension was stirred for $4 \mathrm{~h}$ to evaporate the dichloromethane. The suspension was centrifuged at $12,000 \mathrm{rpm}$ for $15 \mathrm{~min}$ at $4^{\circ} \mathrm{C}$, washed three times, resuspended in the water with cryoprotectant and freeze-dried to obtain the fine FA-LPNs powder.

VCR-loaded FA-decorated LPNs (FA-VCR/LPNs) were fabricated by the same method as method A, with a different 


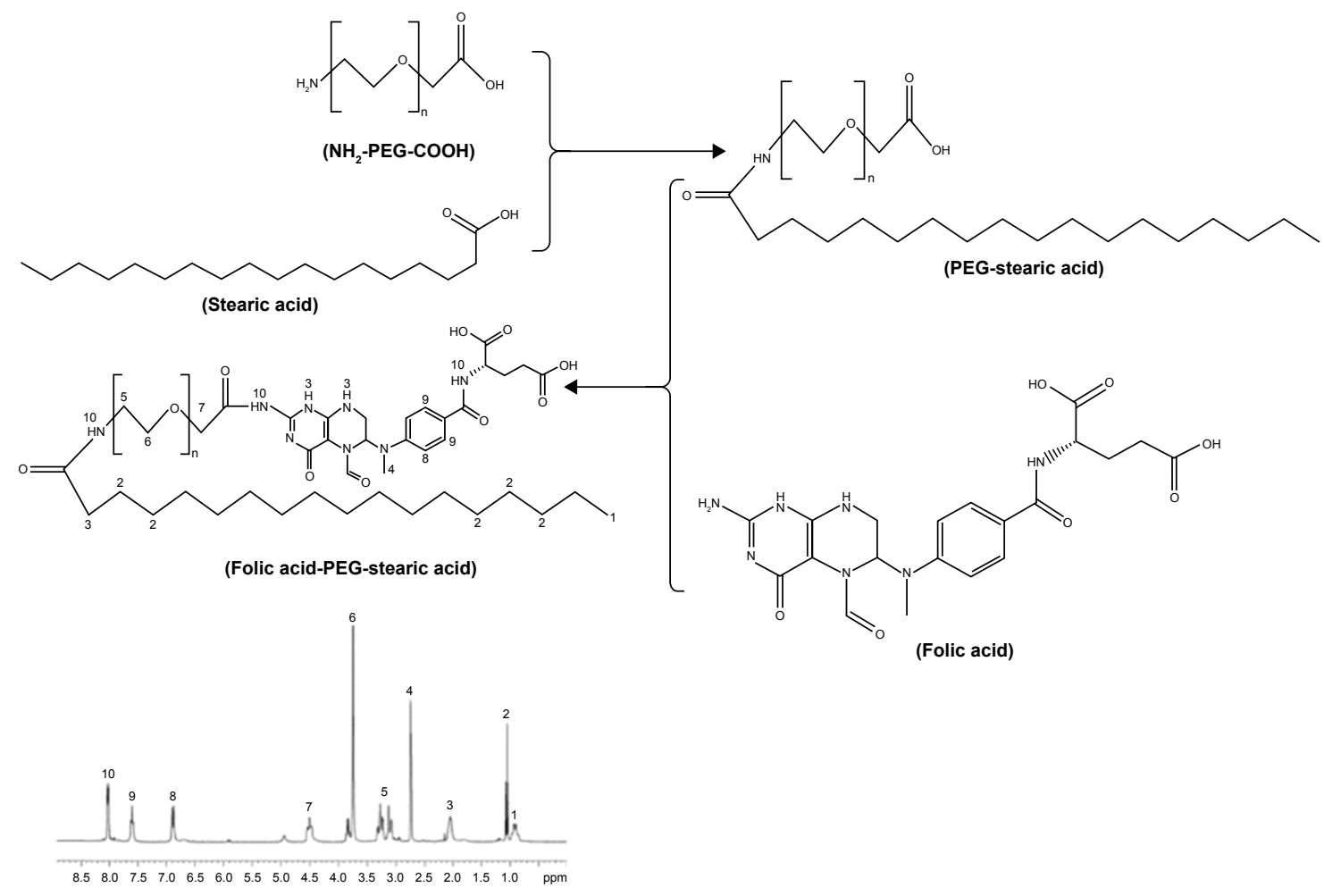

Figure I General reaction scheme and 'H-NMR spectroscopy for FA-decorated lipid (FA-PEG-SA).

Note: Numbers I-10 marked on the 'H-NMR spectroscopy correspond with the numbers marked for the FA-PEG-SA structure.

Abbreviations: NMR, nuclear magnetic resonance; FA, folic acid; PEG, polyethylene glycol; SA, stearic acid.

oil phase: the oil phase was prepared by dissolving VCR and PLGA in dichloromethane (method B) (Figure 2).

VCR-loaded LPNs which do not contain FA(VCR/LPNs) were fabricated by the same method as method B, with a different aqueous phase: the aqueous phase was prepared by dispersing PEG-SA in ultrapure water, by sonication.

\section{Characterization of LPNs}

\section{Average size and surface charge}

Average particle size, size distribution and zeta potential of the LPNs were tested at $\mathrm{pH} 7.4$ and at $25^{\circ} \mathrm{C}$, using a Malvern zetasizer NS (Malvern Instruments, Malvern, UK).

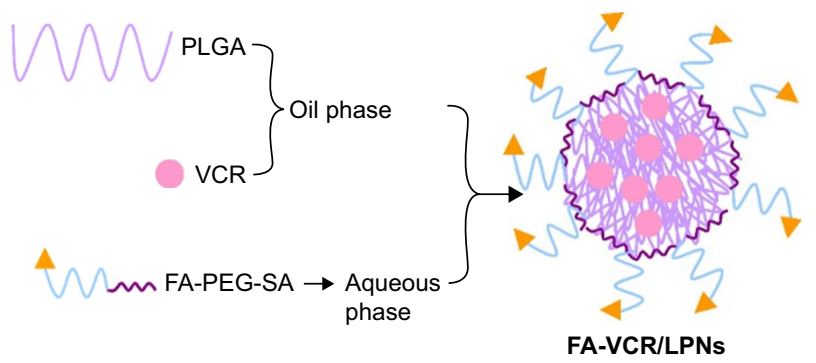

Figure 2 Fabrication of VCR-loaded FA-decorated LPNs (FA-VCR/LPNs). Abbreviations: PLGA, poly(lactic-co-glycolic acid); PEG, polyethylene glycol; VCR, vincristine; FA, folic acid; LPNs, lipid-polymer hybrid nanoparticles.

\section{Drug encapsulation and loading efficiency}

Drug encapsulation efficiency (EE) was determined by measuring the free drug present in the supernatant and comparing it to the total amount of drug added:21

$$
\mathrm{EE}=\frac{\text { Total amount drug }- \text { Amount free drug }}{\text { Total amount drug }} \times 100 \%(1)
$$

Drug loading efficiency (DL) was calculated based on the amount of encapsulated drug and amount of LPNs:

$$
\mathrm{DL}=\frac{\text { Amount of drug in LPNs }}{\text { Amount of LPNs }} \times 100 \%
$$

The amount of encapsulated drug was determined by High Performance Liquid Chromatography (HPLC). ${ }^{19}$

\section{Cellular uptake}

To evaluate cellular uptake efficiency of LPNs, coumarin 6 was used as a model fluorescent molecule, which can be encapsulated into various LPNs for quantitative investigation on cellular uptake. ${ }^{22}$ The Raji/VCR cells were equilibrated with Hank's buffered salt solution (HBSS) at $37^{\circ} \mathrm{C}$ for $1 \mathrm{~h}$ before coumarin 6-loaded LPNs were added at concentrations 
of $200 \mathrm{mg} / \mathrm{mL}$. After incubation for 2, 4, and $8 \mathrm{~h}$, the medium was removed and the wells were washed three times with cold PBS solution. Then, cells were washed once with $1 \mathrm{~mL}$ of PBS and were detached with trypsin/EDTA. Then, the cells were centrifuged at $1,500 \mathrm{rpm}$ at $4{ }^{\circ} \mathrm{C}$ for $5 \mathrm{~min}$, the supernatant was discarded, and the cells were re-suspended in $300 \mu \mathrm{L}$ of PBS and directly introduced to a BD FACSCalibur flow cytometer (BD, Franklin Lakes, NJ, USA).

\section{Determination of folic acid receptor (FR)-expression} levels of cells cultured in vitro

Western blot analyses were carried out for FR confirmation in the KB, Raji, A20 cells, and HUVEC. 48 h-incubated cells were harvested, centrifuged at 2,000 $\mathrm{rpm}\left(4^{\circ} \mathrm{C}\right)$ for $10 \mathrm{~min}$ and washed in cold PBS. Following another centrifugation, at $6,000 \mathrm{rpm}\left(4^{\circ} \mathrm{C}\right)$ for $3 \mathrm{~min}$, cell pellets were resuspended, homogenized in $100 \mu \mathrm{L}$ of ice-cooled radioimmunoprecipitation (RIPA) buffer (1\% NP-40 in $150 \mathrm{mM} \mathrm{NaCl}, 50 \mathrm{mM}$ Tris- $\mathrm{HCl} \mathrm{pH}$ 7.5, $2 \mathrm{mM}$ EDTA), and then incubated for $10 \mathrm{~min}$. The proteins were cleared by centrifugation at $10,000 \mathrm{rpm}\left(4^{\circ} \mathrm{C}\right)$ for $10 \mathrm{~min}$. Western blot was performed with cell lysates ( $\sim 40 \mu$ protein) using anti-FR antibody. Western blot signal was quantified using ImageJ software (version 1.51k; NIH, Rockville, MD, USA). Region of interest (ROI) was chosen manually, based on the largest band in the blot. The same ROI was applied in all remaining rows, with the protein band in the middle of the ROI frame. The mean signal of each ROI was standardized to the signal of $\mathrm{KB}$ cells, which was set as $100 \%{ }^{23}$

\section{In vitro cytotoxicity}

In vitro cytotoxicity of LPNs in Raji cells, Raji/VCR cells, A20 cell line, and HUVEC was evaluated by MTT assay. ${ }^{24}$ Different cells were cultured in $10 \%(\mathrm{v} / \mathrm{v})$ of fetal bovine serum (FBS). MTT assays were performed on Raji cells, Raji/VCR cells and HUVEC upon treatment with free VCR, FA-LPNs, VCR/LPNs, and FA-VCR/LPNs with varying concentrations ranging from $0.1 \mu \mathrm{g} / \mathrm{mL}$ to $100 \mu \mathrm{g} / \mathrm{mL}$ over a period of 72 hours. A microplate spectrophotometer was used to determine the absorbance at $570 \mathrm{~nm}$.

\section{In vivo tissue distribution}

In vivo tissue distribution behavior was evaluated on Raji/ VCR cell bearing BALB/c nude mice. ${ }^{25}$ Free VCR, VCR/ LPNs, and FA-VCR/LPNs (contained $10 \mathrm{mg} / \mathrm{kg}$ of VCR) were intravenously injected via the tail vein, respectively. At determined times (10 min, 1 h, 2 h, 4 h, 8 h, 24 h, 48 h and 72 h) after intravenous injection, following anesthesia with a lethal dose of pentobarbital injected intraperitoneally, the tissues including the tumor, heart, liver, spleen, lungs, and kidneys were dissected, rinsed with $0.9 \% \mathrm{NaCl}$ and blotted using tissue paper to remove adherent blood and fatty matter. Approximately 50-100 mg of the tissues were weighed and homogenized. The amount of drugs was quantified by HPLC using the method mentioned in the "Drug encapsulation and loading efficiency" section.

\section{In vivo pharmacokinetics studies}

The pharmacokinetics of LPNs were investigated using BALB/c nude mice. Free VCR, VCR/LPNs, and FA-VCR/ LPNs (each contained $10 \mathrm{mg} / \mathrm{kg}$ of VCR) were intravenous injected via the tail vein, respectively. ${ }^{26}$ Blood samples $(300 \mu \mathrm{L})$ were collected at pre-determined times $(0.25,0.5$, $0.75,1,2,4,6,8,12,24,48$, and $72 \mathrm{~h}$ ) in polypropylene microcentrifuge tubes, centrifuged at 15,000 rpm for 5 minutes to isolate the plasma, and stored at $-80^{\circ} \mathrm{C}$ and subsequently analyzed by HPLC using the method mentioned in the "Drug encapsulation and loading efficiency" section.

\section{In vivo antitumor activity}

In vivo antitumor activity was evaluated on Raji/VCR cell bearing BALB/c nude mice. ${ }^{27}$ Mice were randomly divided into five groups, each group having six mice $(n=6)$. Each mouse was treated with PBS (control), FA/LPNs, free VCR, VCR/LPNs, and FA-VCR/LPNs (each contained $10 \mathrm{mg} / \mathrm{kg}$ of VCR) intravenously twice a week for 3 weeks. Tumor size was measured twice a week and tumor volumes (TV) were estimated using the following equation:

$$
\mathrm{TV}=\frac{{\text { Length } \times \mathrm{Width}^{2}}^{2}}{2}
$$

The tumor weight (TW) was also assessed. Tumor inhibition efficiency (TIE) calculated through TW was according to the following equation:

$$
\mathrm{TIE}=\frac{\mathrm{TW}_{\text {control group }}-\mathrm{TW}_{\text {treated group }}}{\mathrm{TW}_{\text {control group }}} \times 100 \%
$$

To evaluate the systemic toxicity, body weight was measured and investigated.

\section{Statistical analysis}

Data were expressed as the means \pm standard deviation (SD). Statistical analysis was performed by Student's unpaired $t$-test or one-way analysis of variance (ANOVA) to identify 
significant differences, unless otherwise indicated. Differences were considered significant at $P<0.05$.

\section{Results}

\section{Synthesis and characterization of FA-PEG-SA}

FA-PEG-SA was synthesized by the reaction between the carboxy and amino groups of FA, PEG, and SA. ${ }^{1} \mathrm{H}-\mathrm{NMR}$ spectroscopy was presented one to one corresponding to the FA-PEG-SA structure (Figure 1). The peaks of 3, 7, and 10 proved the formation of the amido bond. Other peaks illustrate the presence of FA, PEG, and SA. The production rate was $78 \%$.

\section{Characterization of LPNs}

The particle size and other physicochemical characteristics of the LPNs' formulations are presented in Table 1. The particle size of FA-VCR/LPNs was $174.1 \mathrm{~nm}$, with a polydispersity index of 0.182 . The size and polydispersity index of FA-LPNs and VCR/LPNs showed no obvious difference with FA-VCR/LPNs. The zeta potential of FA-LPNs, VCR/LPNs, and FA-VCR/LPNs was $-30.6,-35.4$ and $-32.7 \mathrm{mV}$, respectively. The EEs of drugs encapsulated in LPNs were all over $90 \%$. The DL of VCR in VCR/LPNs, and FA-VCR/LPNs was 5.3 and $4.8 \%$.

\section{Cellular uptake}

Cellular uptake efficiency of the coumarin 6-loaded LPNs was tested (Figure 3). As shown in this figure, cellular uptake efficiency of FA-VCR/LPNs and FA-LPNs was significantly higher than VCR/LPNs $(P<0.05)$. Cellular uptake efficiency of FA-VCR/LPNs reached $73.7 \%$ at $8 \mathrm{~h}$ posttreatments.

\section{Determination of FR-expression levels of cells cultured in vitro}

Western blot technique was used to determine relative FR-expression levels in all cancer cell lines (Figure 4).

Table I Characterization of LPNs (mean \pm SD, $n=3$ )

\begin{tabular}{llll}
\hline LPN & FA-LPNs & VCR/LPNs & FA-VCR/LPNs \\
formulations & & & \\
\hline Particle size $(\mathrm{nm})$ & $168.7 \pm 4.6$ & $171.2 \pm 5.2$ & $174.1 \pm 5.5$ \\
Polydispersity index & $0.152 \pm 0.019$ & $0.169 \pm 0.021$ & $0.182 \pm 0.030$ \\
Zeta potential $(\mathrm{mV})$ & $-30.6 \pm 2.7$ & $-35.4 \pm 3.1$ & $-32.7 \pm 3.7$ \\
VCR EE (\%) & $\mathrm{N} / \mathrm{A}$ & $93.2 \pm 2.9$ & $91.4 \pm 3.2$ \\
VCR DL (\%) & $\mathrm{N} / \mathrm{A}$ & $5.3 \pm 0.8$ & $4.8 \pm 0.7$ \\
\hline
\end{tabular}

Abbreviations: LPNs, lipid-polymer hybrid nanoparticles; FA-LPNs, folic aciddecorated LPNs; VCR, vincristine; VCR/LPNs, VCR-loaded LPNs; FA-VCR/LPNs, folic acid-decorated VCR-loaded LPNs; EE, drug encapsulation efficiency; DL, drug loading efficiency; N/A, not applicable.

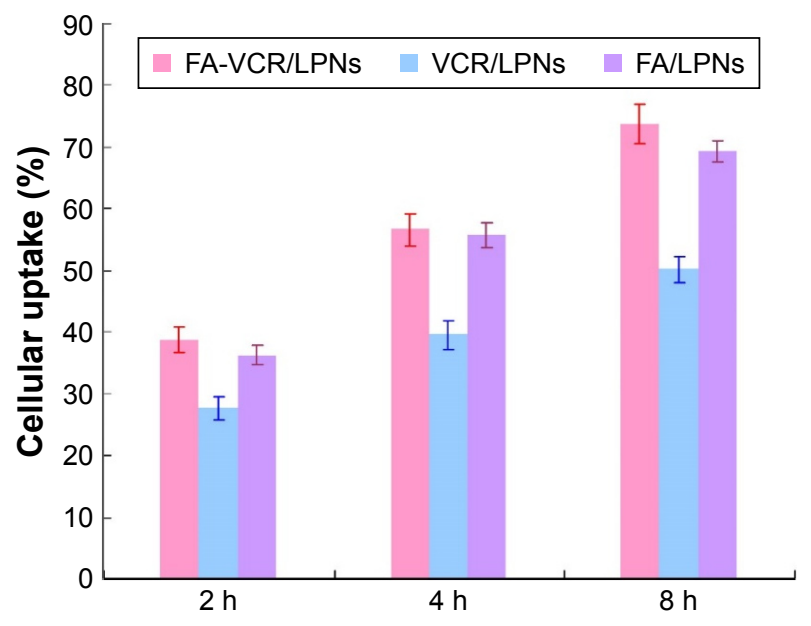

Figure 3 Cellular uptake efficiency of the coumarin 6-loaded LPNs. Abbreviations: VCR, vincristine; FA, folic acid; LPNs, lipid-polymer hybrid nanoparticles.

Among the tested cervical cancer cell lines, $\mathrm{KB}$ cells revealed the most prominent FR-expression, followed by Raji cells and A20 cell line. HUVEC showed very low FR-expression. FR-expression was higher in Raji cells than A20 cell line, so Raji cells were chosen for the subsequent experiments.

\section{In vitro cytotoxicity}

In vitro cytotoxicity of different formulations was illustrated as the cell viability of the treated Raji, Raji/VCR cells, and A20 cell line (Figure 5). It can be seen from the figure that all the formulations complied with a concentration-dependent pattern; the cytotoxicity increased along with the increase of the drug concentrations. FA-VCR/LPNs exhibited higher cell toxicity than undecorated VCR/LPNs $(P<0.05)$. VCR-encapsulated LPNs achieved better efficiency to defeat cancer cells than free VCR $(P<0.05)$. Free VCR exhibited higher cytotoxicity in Raji cells than Raji/VCR cells. FA-LPNs do not contain drugs and showed no obvious toxicity; cell viability was over $80 \%$. LPNs showed lower toxicity in HUVEC.

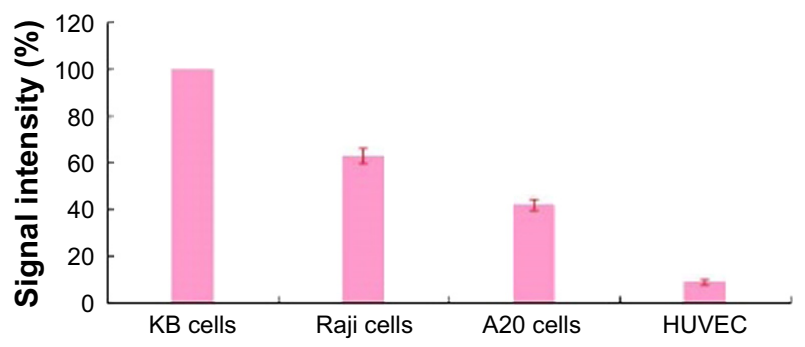

Figure 4 Quantification of signal intensity obtained from Western blot for FRexpression in KB cells, Raji cells and A20 cell line. The value obtained for KB cells was set as $100 \%$.

Abbreviations: FR, folic acid receptor; HUVEC, human umbilical vein endothelial cells. 

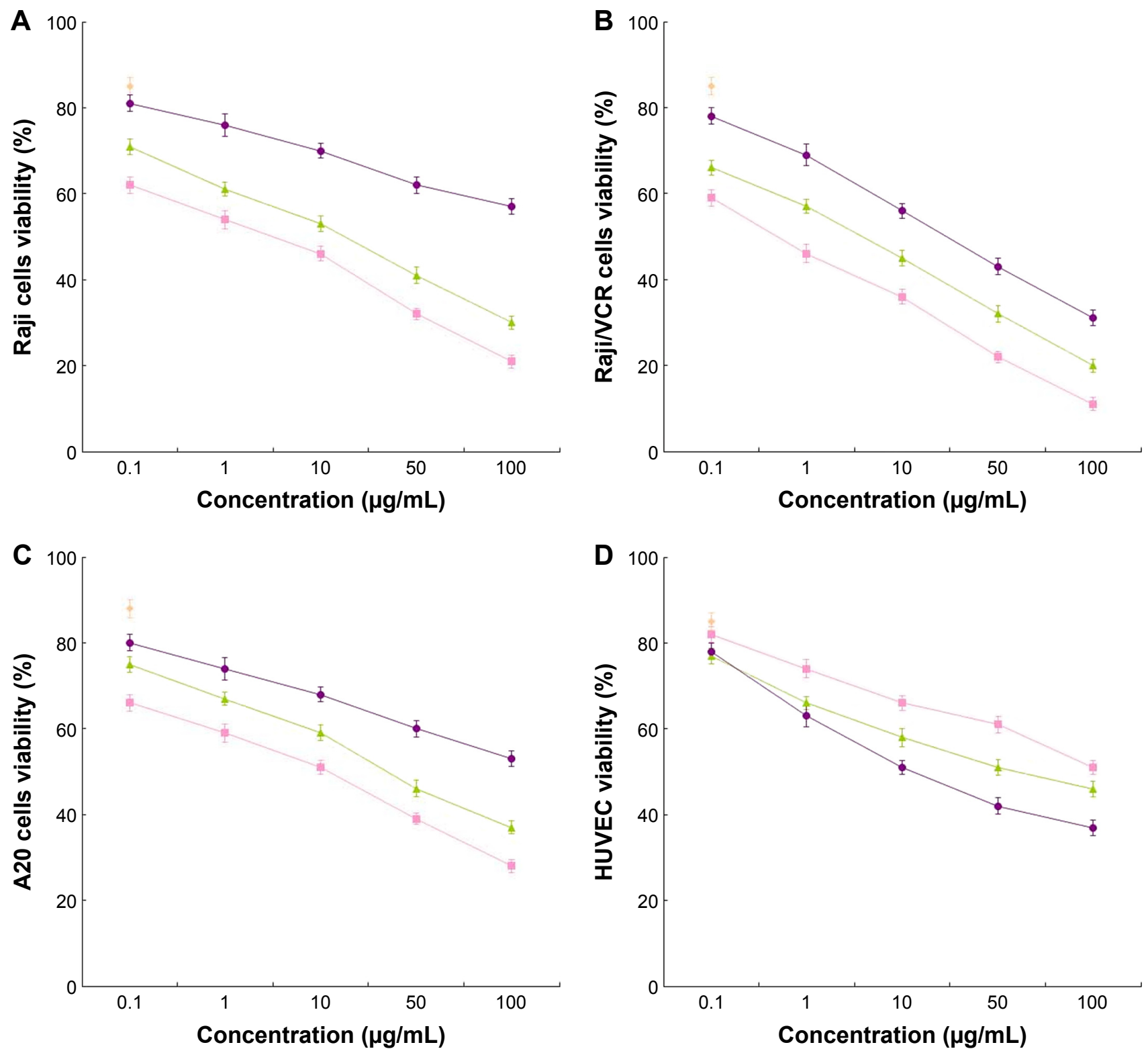

$$
\text { -FA-VCR/LPNs } \rightarrow \text { VCR/LPNs } \quad \text { FA-LPNs } \rightarrow \text { Free VCR }
$$

Figure $\mathbf{5}$ In vitro cytotoxicity of different formulations illustrated as the cell viability of the treated Raji cells (A), Raji/VCR cells (B), A20 cells (C), and HUVEC cells (D). Notes: In Raji/VCR cells, FA-VCR/LPNs exhibited higher cell toxicity than undecorated VCR/LPNs. VCR-encapsulated LPNs achieved better efficiency to defeat cancer cells than free VCR. FA-LPNs do not contain drugs and showed no obvious toxicity. Data represent the mean $\pm S D(n=3)$.

Abbreviations: VCR, vincristine; LPNs, lipid-polymer hybrid nanoparticles; FA, folic acid; HUVEC, human umbilical vein endothelial cells; FA-VCR/LPNs, folic acid-decorated VCR-loaded LPNs; FA-LPNs, folic acid-decorated LPNs; VCR/LPNs, VCR-loaded LPNs.

\section{In vivo tissue distribution}

In vivo tissue distribution of $\mathrm{VCR} / \mathrm{LPNs}$, and $\mathrm{FA}-\mathrm{VCR} /$ LPNs was investigated in Raji/VCR cells in mice after intravenous injection and compared to the distribution of free VCR (Figure 6). In summary, drug distribution of free VCR rapidly reached a high level during the first few hours and reduced over time. VCR distribution of VCR/LPNs and FA-VCR/LPNs was significantly higher in tumor tissue than in the free $\operatorname{VCR}(P<0.05)$. The opposite results were found in the heart where a lower distribution of VCR was found in VCR/LPNs and FA-VCR/LPNs groups than in the free VCR group $(P<0.05)$.

\section{In vivo pharmacokinetics}

The plasma concentration of VCR after the administration of free VCR, VCR/LPNs, and FA-VCR/LPNs is shown in Figure 7 and the pharmacokinetics' parameters such as area under the curve (AUC), plasma elimination half-life $\left(t_{1 / 2}\right)$, 

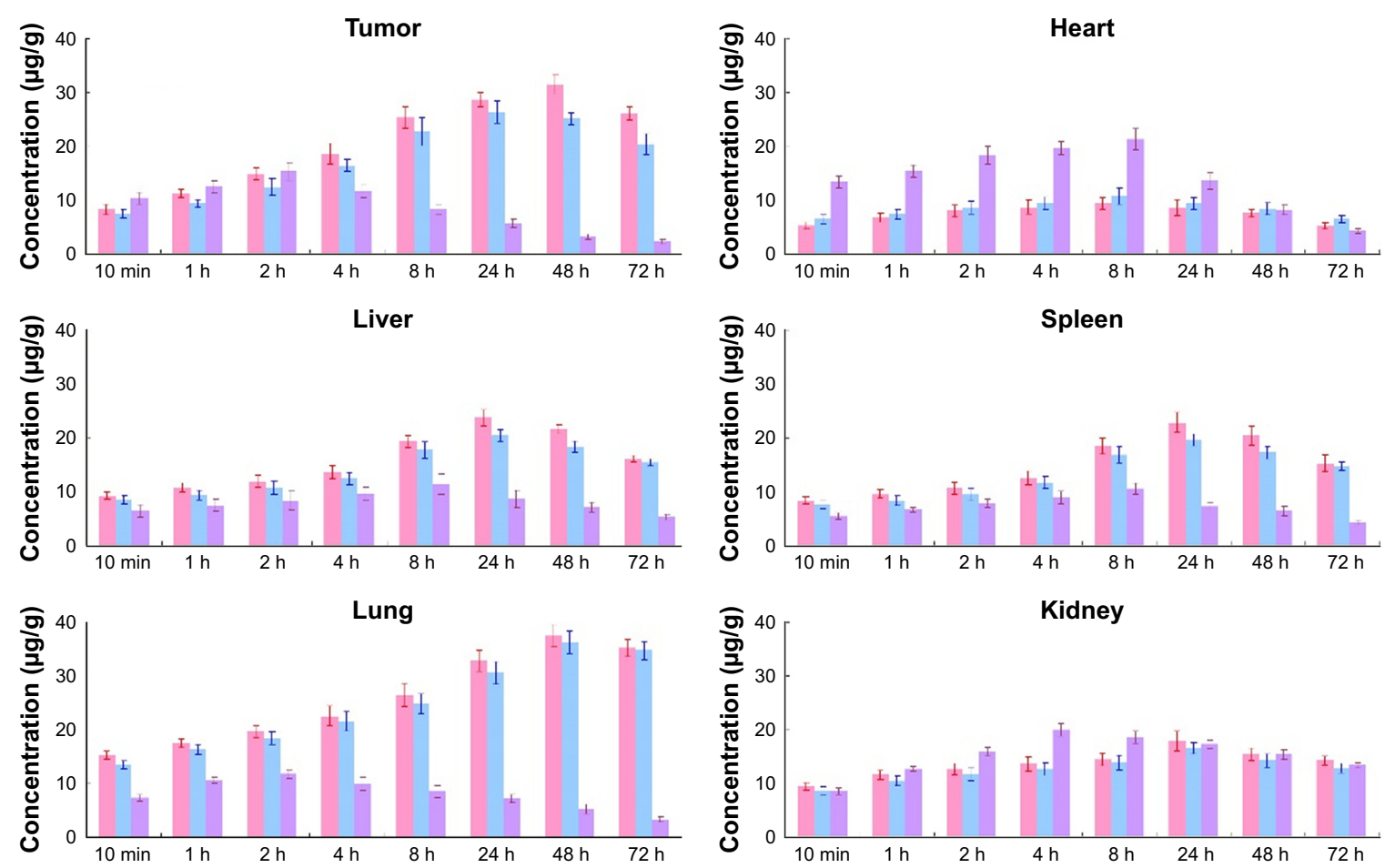

$\square$ FA-VCR/LPNs $\square$ VCR/LPNs $\square$ Free VCR

Figure 6 In vivo tissue distribution of free VCR, VCR/LPNs, and FA-VCR/LPNs investigated in Raji/VCR cell bearing mice after intravenous injection.

Notes: VCR distribution of VCR/LPNs and FA-VCR/LPNs was significantly higher in tumor tissue than the free VCR. Drug distribution of free VCR rapidly reached a high level during the first few hours and reduced over time. Data represent the mean $\pm S D(n=6)$.

Abbreviations: VCR, vincristine; LPNs, lipid-polymer hybrid nanoparticles; FA, folic acid; FA-VCR/LPNs, folic acid-decorated VCR-loaded LPNs; VCR/LPNs, VCR-loaded LPNs.

and mean residence time (MRT) were calculated and presented in Table 2. The AUC, MRT, and $t_{1 / 2}$ of FA-VCR/ LPNs were $377.24 \mu \mathrm{g} \cdot \mathrm{h} / \mathrm{mL}, 10.32 \mathrm{~h}$, and $6.94 \mathrm{~h}$, respectively, which were significantly higher than that of VCR/ LPNs and free VCR $(P<0.05)$.

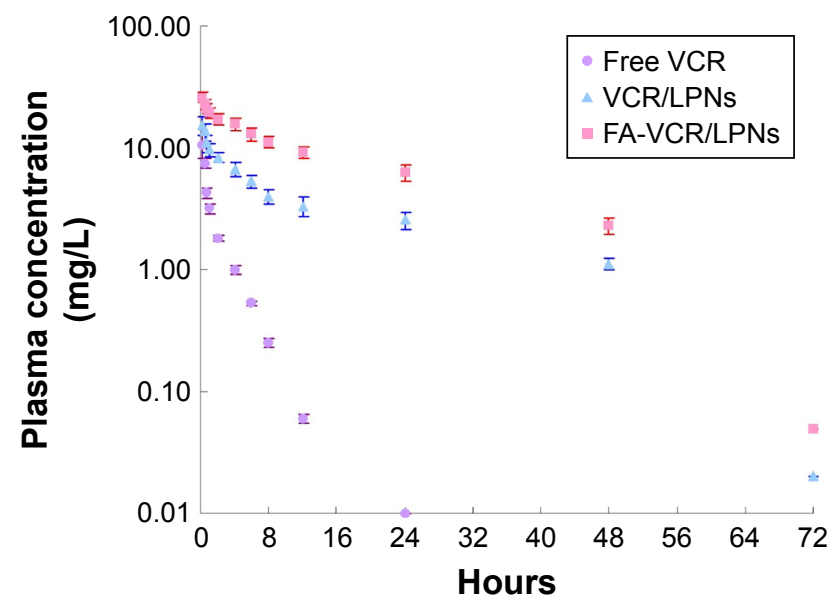

Figure 7 The plasma concentration of VCR after the administration of free VCR, VCR/LPNs, and FA-VCR/LPNs.

Note: Data represent the mean \pm SD $(n=6)$.

Abbreviations: VCR, vincristine; LPNs, lipid-polymer hybrid nanoparticles; FA, folic acid; VCR/LPNs, VCR-loaded LPNs; FA-VCR/LPNs, folic acid-decorated VCRloaded LPNs.

\section{In vivo antitumor activity}

In vivo antitumor activity of LPNs was evaluated along with the free drug (Figure 7). Tumor volume increased rapidly when the mice were treated with PBS or FA-LPNs. The tumor volumes of all the drug-containing groups were inhibited more than in the PBS group (Figure 8A). The tumors of mice treated with FA-VCR/LPNs were considerably smaller than the VCR/LPNs and the free VCR groups $(P<0.05)$. After 3 weeks of administration, the TIE and body weight

Table 2 Pharmacokinetic parameters of VCR after intravenous administration of formulations to mice (mean $\pm S D, n=6$ )

\begin{tabular}{llll}
\hline $\begin{array}{l}\text { Pharmacokinetic } \\
\text { parameters }\end{array}$ & $\begin{array}{l}\text { Free } \\
\text { VCR }\end{array}$ & VCR/LPNs & FA-VCR/LPNs \\
\hline AUC $_{(0 \rightarrow 72)}(\mu \mathrm{g} \cdot \mathrm{h} / \mathrm{mL})$ & $18.29 \pm 2.08$ & $346.43 \pm 23.28^{*}$ & $377.24 \pm 26.17^{*}$ \\
$\mathrm{AUC} \mathrm{(}_{(0 \rightarrow \infty)}(\mu \mathrm{g} \cdot \mathrm{h} / \mathrm{mL})$ & $19.34 \pm 2.34$ & $387.21 \pm 31.19^{*}$ & $412.36 \pm 33.25^{*}$ \\
$\mathrm{MRT}^{(\mathrm{h})}$ & $2.89 \pm 0.36$ & $8.98 \pm 0.76^{*}$ & $10.32 \pm 0.95^{*}$ \\
$t_{1 / 2}(\mathrm{~h})$ & $1.88 \pm 0.27$ & $5.86 \pm 0.67^{*}$ & $6.94 \pm 0.52^{*}$ \\
$K_{\mathrm{e}}\left(\mathrm{h}^{-1}\right)$ & $0.43 \pm 0.06$ & $0.21 \pm 0.04^{*}$ & $0.16 \pm 0.03^{*}$ \\
\hline
\end{tabular}

Note: $* P<0.05$ compared with free VCR.

Abbreviations: LPNs, Lipid-polymer hybrid nanoparticles; FA-LPNs, folic aciddecorated LPNs; VCR, vincristine; VCR/LPNs, VCR-loaded LPNs; FA-VCR/LPNs, folic acid-decorated VCR-loaded LPNs; AUC, area under the curve; MRT, mean residence time; $\mathrm{t}_{1 / 2}$, half-life; $\mathrm{K}_{\mathrm{e}}$, terminal elimination rate. 

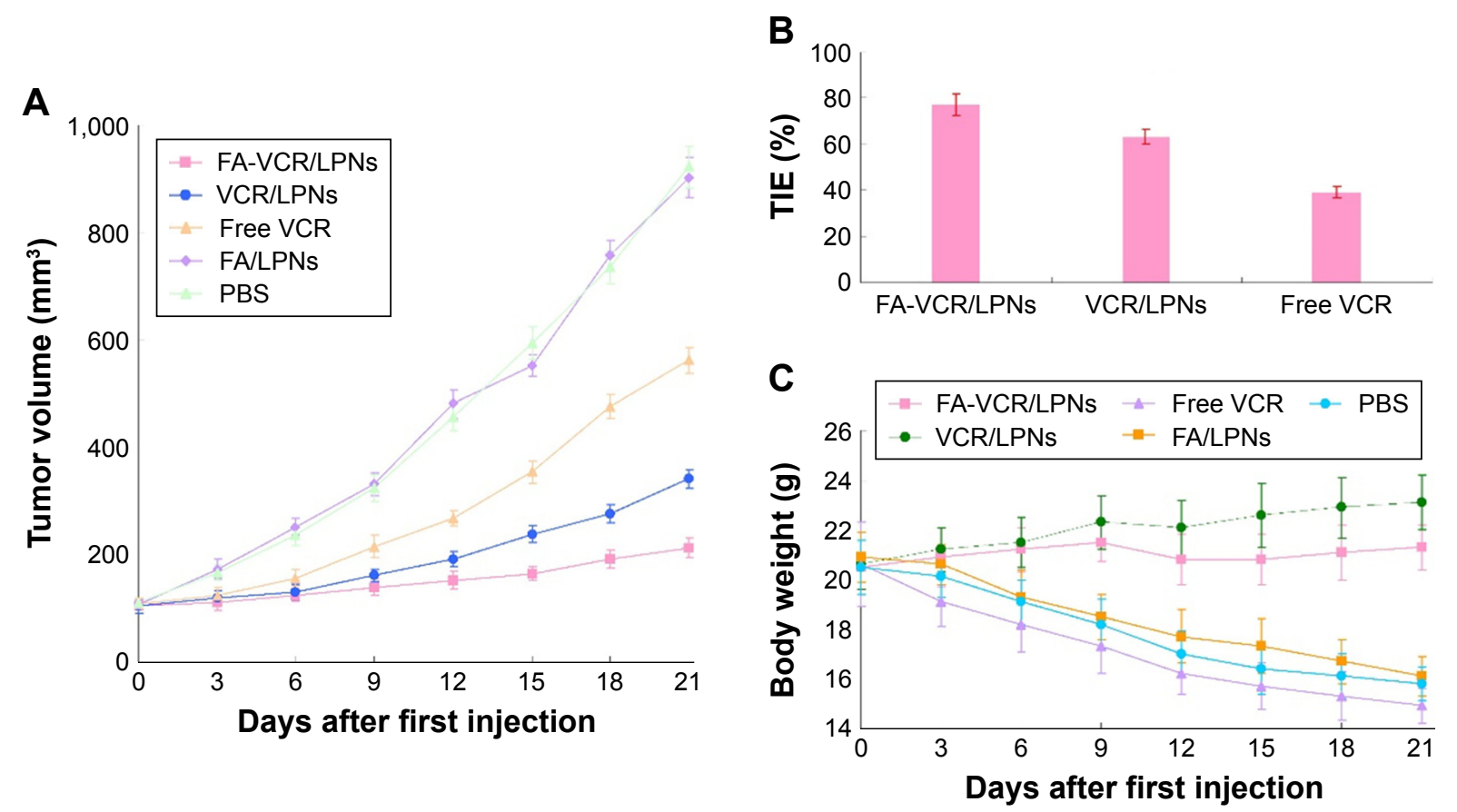

Figure 8 In vivo antitumor activity of LPNs was evaluated along with the free drug: tumor volume (A); TIE (B); and body weight (C).

Notes: Tumor volume increased rapidly when the mice were treated with PBS or FA-LPNs. The tumor volumes of all the drug-containing groups were more inhibited than the PBS group. The tumors of mice treated with FA-VCR/LPNs were considerably smaller than VCR/LPNs and free VCR groups. Data represent the mean \pm SD ( $n=6$ ).

Abbreviations: VCR, vincristine; LPNs, lipid-polymer hybrid nanoparticles; FA, folic acid; TIE, tumor inhibition efficiency; VCR/LPNs, VCR-loaded LPNs; FA-VCR/LPNs, folic acid-decorated VCR-loaded LPNs; FA-LPNs, folic acid-decorated LPNs.

of tumor-bearing mice were summarized. FA-VCR/LPNs exhibited the highest TIE (Figure 8B). No significant weight loss was observed in any of the VCR-loaded LPNs' groups. However, reduction in body weight was observed in the free drug solution and FA-LPNs groups (Figure 8C).

\section{Discussion}

FA-decorated LPNs loading anticancer drugs have been developed in this study for the treatment of folate receptor positive lymphoma. Since FA is a crucial vitamin for proliferating cells, namely cancer cells, it would promote drug delivery by mediating internalization of FA-containing lipoplexes, most likely by FA receptor mediated-endocytosis. ${ }^{28}$ The choice of this ligand is very attractive because of its high binding affinity, low immunogenicity, ease of modification, small size, stability during storage, and low cost. Firstly, FA-PEG-SA was synthesized by the reaction between the carboxy and amino groups of FA, PEG, and SA. The nature and concentration of the targeting ligand is a key variable in ligand-mediated cell targeting. ${ }^{16}$ The differences reported between the various ligands in optimal concentrations for receptor binding may be related to the accessibility of the folate moiety of the ligand, which may be related to the PEG length or to the PEG-folate chemical linkage. $\mathrm{PEG}_{5000}$, the space linker with longer chain length has been proven to have better recognition of the targeting moiety on the NPs surface by the receptors. ${ }^{29}$ In this study, $\mathrm{NH}_{2}-\mathrm{PEG}_{5000}{ }^{-}$ $\mathrm{COOH}$ was utilized. Then FA-LPNs with polymeric PLGA cores and lipid shells were fabricated by the ultrasonication method. The particle size of FA/LPNs, VCR/LPNs and FA-VCR/LPNs was around $170 \mathrm{~nm}$, indicating that loading of the drug did not increase the diameter of the LPNs. The polydispersity of LPNs was below 0.2 , which is not a wide size distribution. The drug EE of the NPs is crucial to justify their clinical applications. The EEs of drugs encapsulated in LPNs were all over $90 \%$.

The cellular uptake research could provide some circumstantial evidence to display the advantages of the LPNs formulation. Coumarin 6, a fluorescent probe, was used to represent the drug in the nanoparticle formulation to analyze cellular uptake efficiency. Cellular uptake efficiency of FAdecorated LPNs was significantly higher than VCR/LPNs. This could be attributed to enhanced cancer cell specific adherence of the FA ligands. ${ }^{22}$ In vitro cytotoxicity studies were initially conducted with empty FA-LPNs to determine the vehicle cytotoxicity. ${ }^{30}$ A number of human gynecological cancer cell lines are known, or mentioned in the literature, to express FR..$^{23}$ The use of the cervical adenocarcinoma cells is most common in the field, due to the very high FR-expression level in KB cells. KB cells are a subclone of 
HeLa cells, which was the first human epithelial cancer cell line established in culture and probably the best-known cell line in past and current research. Western blot technique was used to determine relative FR-expression levels in all cancer cell lines. HUVEC showed very low FR-expression. FR-expression was higher in Raji cells than A20 cell line, so Raji cells were chosen for the subsequent experiments.

Cell viability of FA-LPNs was over $80 \%$, thus showing no observable toxicity. The most noteworthy enhancement of VCR cytotoxicity was observed in the FA-VCR/LPNs group in Raji and Raji/VCR cells. This indicated the importance of encapsulation of anticancer agents in FA-decorated LPNs. VCRencapsulated LPNs achieved better efficiency to defeat cancer cells than free VCR illustrating the excellent lymph tumor cell inhibition ability of the resulting LPN formulas, accounting for the outstanding antitumor activity in vitro. In HUVEC, LPNs showed lower toxicity than in cancer cells, indicating the targeted ability of LPNs to Raji and Raji/VCR cells.

High percentages of tissue distribution and penetration into target tissues are critical for an effective system. ${ }^{31}$ In vivo tissue distribution behavior of LPNs was evaluated on Raji/VCR cell bearing BALB/c nude mice. In summary, drug distribution of free VCR rapidly reached a high level during the first few hours and reduced over time. VCR distribution of VCR/LPNs and FA-VCR/LPNs was significantly higher in tumor tissue than the free VCR. The opposite results were found in the heart; obviously lower distribution of VCR was found in VCR/LPNs and FA-VCR/LPNs groups than free VCR group. Systemic toxicity could be decreased if fewer drug distributions in the heart and kidney could be achieved. Higher tumor tissue distribution may lessen the side effects. ${ }^{19}$ Noticeably, a higher accumulation of the VCR-loaded LPNs was noted in the lung and spleen than free VCR, which may cause some side effects.

Nanoparticulate drug delivery systems are proved to have the ability to alter the pharmacokinetic profiles of drugs. ${ }^{32}$ This study illustrated that LPNs and free drugs have different pharmacokinetic properties. The relatively higher initial plasma concentration and longer drug retention of LPNs suggest that LPNs might be promising systems to reduce the elimination of the VCR by the mononuclear phagocyte system and extend the residence time of the VCR in the circulatory system. FA-VCR/LPNs showed a higher AUC than those of VCR/LPNs and free VCR, and it was expected that FA-VCR/LPNs could extend its in vivo antitumor activity.

To determine whether FA-VCR/LPNs are able to improve the therapeutic efficacy on lymphoma in vivo, VCR-encapsulated LPNs and free VCR were evaluated separately on lymphoma-bearing BALB/c nude mice. Results showed that free VCR had some effect on inhibiting tumor growth and was not statistically different from the negative control. ${ }^{33}$ The effect of the encapsulation of drugs as a strategy to improve the in vivo anticancer efficacy was evident for VCR, and significant antitumor activity was observed. No significant weight loss was observed in any of the VCR-loaded LPNs groups, indicating that all the treatments were well tolerated. However, reduction in body weight was observed in the free drug groups. The reduction of body weight with FA-LPNs may be caused by the increase of tumor progression that made the mice reduce their food intake and lose weight, as with the PBS control group. However, FA-LPNs do not contain drugs and showed no obvious toxicity during the cell viability study. Because of these results, LPNs constructed in this research can be regarded as one of the most biocompatible and biosafe delivery systems, and could be applied for the treatment of many diseases, particularly cancer.

\section{Conclusion}

This study reveals that the FA-decorated LPNs system can not only deliver therapeutic drugs in targeting tumor tissues but also has the capability to promote in vivo antitumor effects. The resulting FA-VCR/LPNs could be an effective and practical approach to enhance the efficacy of antilymphoma agent and could be developed further to achieve better therapeutic effects.

\section{Disclosure}

The authors report no conflicts of interest in this work.

\section{References}

1. Knapp CM, Whitehead KA. In pursuit of a moving target: nanotherapeutics for the treatment of non-Hodgkin B-cell lymphoma. Expert Opin Drug Deliv. 2014;11(12):1923-1937.

2. Li H, Guo K, Wu C, et al. Controlled and targeted drug delivery by a UVresponsive liposome for overcoming chemo-resistance in non-Hodgkin lymphoma. Chem Biol Drug Des. 2015;86(4):783-794.

3. Shankland KR, Armitage JO, Hancock BW. Non-Hodgkin lymphoma. Lancet. 2012;380(9844):848-857.

4. Barenholz Y. Doxil ${ }^{\circledR}$ - the first FDA approved nano-drug: lessons learned. J Control Release. 2012;160(2):117-134.

5. Gabizon A, Catane R, Uziely B, et al. Prolonged circulation time and enhanced accumulation in malignant exudates of doxorubicin encapsulated in polyethylene-glycol coated liposomes. Cancer Res. 1994;54(4): 987-992.

6. Chen WC, Completo GC, Sigal DS, Crocker PR, Saven A, Paulson JC. In vivo targeting of B-cell lymphoma with glycan ligands of CD22. Blood. 2010;115(23):4778-4786.

7. Kato J, Li Y, Xiao K, et al. Disulfide crosslinked micelles for the targeted delivery of vincristine to B-cell lymphoma. Mol Pharm. 2012;9(6): $1727-1735$ 
8. Park J, Fong PM, Lu J, et al. PEGylated PLGA nanoparticles for the improved delivery of doxorubicin. Nanomedicine. 2009;5(4):410-418.

9. Xiao K, Luo J, Li Y, Lee JS, Fung G, Lam KS. PEG-oligocholic acid telodendrimer micelles for the targeted delivery of doxorubicin to B-cell lymphoma. J Control Release. 2011;155(2):272-281.

10. Hadinoto K, Sundaresan A, Cheow WS. Lipid-polymer hybrid nanoparticles as a new generation therapeutic delivery platform: a review. Eur J Pharm Biopharm. 2013;85(3):427-443.

11. Li Y, Wu H, Yang X, et al. Mitomycin C-soybean phosphatidylcholine complex-loaded self-assembled PEG-lipid-PLA hybrid nanoparticles for targeted drug delivery and dual-controlled drug release. Mol Pharm. 2014;11(8):2915-2927.

12. Salvador-Morales C, Zhang L, Langer R, Farokhzad OC. Immunocompatibility properties of lipid-polymer hybrid nanoparticles with heterogeneous surface functional groups. Biomaterials. 2009;30(12): 2231-2240

13. Zhang L, Zhu D, Dong X, et al. Folate-modified lipid-polymer hybrid nanoparticles for targeted paclitaxel delivery. Int $J$ Nanomedicine. 2015;10:2101-2114.

14. Berguig GY, Convertine AJ, Shi J, et al. Intracellular delivery and trafficking dynamics of a lymphoma-targeting antibody-polymer conjugate. Mol Pharm. 2012;9(12):3506-3514.

15. Loureiro A, Bernardes GJ, Shimanovich U, et al. Folic acid-tagged protein nanoemulsions loaded with CORM-2 enhance the survival of mice bearing subcutaneous A20 lymphoma tumors. Nanomedicine. 2015; 11(5):1077-1083.

16. Shmeeda H, Mak L, Tzemach D, Astrahan P, Tarshish M, Gabizon A. Intracellular uptake and intracavitary targeting of folate-conjugated liposomes in a mouse lymphoma model with up-regulated folate receptors. Mol Cancer Ther. 2006;5(4):818-824.

17. Tuscano JM, Martin SM, Ma Y, Zamboni W, O’Donnell RT. Efficacy, biodistribution, and pharmacokinetics of CD22-targeted pegylated liposomal doxorubicin in a B cell non-Hodgkin's lymphoma xenograft mouse model. Clin Cancer Res. 2010;16(10):2760-2768.

18. Liu Y, He M, Niu M, et al. Delivery of vincristine sulfate-conjugated gold nanoparticles using liposomes: a light-responsive nanocarrier with enhanced antitumor efficiency. Int J Nanomedicine. 2015;10:3081-3095.

19. Ni S, Qiu L, Zhang G, Zhou H, Han Y. Lymph cancer chemotherapy: delivery of doxorubicin-gemcitabine prodrug and vincristine by nanostructured lipid carriers. Int J Nanomedicine. 2017;12:1565-1576.

20. Liu Y, Li K, Pan J, Liu B, Feng SS. Folic acid conjugated nanoparticles of mixed lipid monolayer shell and biodegradable polymer core for targeted delivery of Docetaxel. Biomaterials. 2010;31(2):330-338.
21. Silva CO, Rijo P, Molpeceres J, et al. Polymeric nanoparticles modified with fatty acids encapsulating betamethasone for anti-inflammatory treatment. Int J Pharm. 2015;493(1-2):271-284.

22. Liu B, Han L, Liu J, Han S, Chen Z, Jiang L. Co-delivery of paclitaxel and TOS-cisplatin via TAT-targeted solid lipid nanoparticles with synergistic antitumor activity against cervical cancer. Int J Nanomedicine. 2017;12:955-968

23. Siwowska K, Schmid RM, Cohrs S, Schibli R, Müller C. Folate receptorpositive gynecological cancer cells: in vitro and in vivo characterization. Pharmaceuticals (Basel). 2017;10(3):pii, E72.

24. Joseph MM, Aravind SR, George SK, Pillai RK, Mini S, Sreelekha TT. Co-encapsulation of doxorubicin with galactoxyloglucan nanoparticles for intracellular tumor-targeted delivery in murine ascites and solid tumors. Transl Oncol. 2014;7(5):525-536.

25. Gaudin A, Lepetre-Mouelhi S, Mougin J, et al. Pharmacokinetics, biodistribution and metabolism of squalenoyl adenosine nanoparticles in mice using dual radio-labeling and radio-HPLC analysis. $J$ Control Release. 2015;212:50-58.

26. Baek JS, Kim JH, Park JS, Cho CW. Modification of paclitaxel-loaded solid lipid nanoparticles with 2-hydroxypropyl- $\beta$-cyclodextrin enhances absorption and reduces nephrotoxicity associated with intravenous injection. Int J Nanomedicine. 2015;10:5397-5405.

27. Kim CE, Lim SK, Kim JS. In vivo antitumor effect of cromolyn in PEGylated liposomes for pancreatic cancer. J Control Release. 2012; 157(2):190-195.

28. Duarte S, Faneca H, de Lima MC. Non-covalent association of folate to lipoplexes: a promising strategy to improve gene delivery in the presence of serum. J Control Release. 2011;149(3):264-272.

29. Pan X, Wang H, Shukla S, et al. Boron-containing folate receptor targeted liposomes as potential delivery agents for neutron capture therapy. Bioconjug Chem. 2002;13(3):435-442.

30. Xu Y, Qiu L. Nonspecifically enhanced therapeutic effects of vincristine on multidrug-resistant cancers when coencapsulated with quinine in liposomes. Int J Nanomedicine. 2015;10:4225-4237.

31. Li Y, Pan WS, Chen SL, Xu HX, Yang DJ, Chan AS. Pharmacokinetic, tissue distribution, and excretion of puerarin and puerarin-phospholipid complex in rats. Drug Dev Ind Pharm. 2006;32(4):413-422.

32. Lou $\mathrm{H}$, Zhang $\mathrm{X}$, Gao $\mathrm{L}$, et al. In vitro and in vivo antitumor activity of oridonin nanosuspension. Int J Pharm. 2009;379(1):181-186.

33. Eloy JO, Petrilli R, Topan JF, et al. Co-loaded paclitaxel/rapamycin liposomes: development, characterization and in vitro and in vivo evaluation for breast cancer therapy. Colloids Surf B Biointerfaces. 2016; 141:74-82.
Drug Design, Development and Therapy

\section{Publish your work in this journal}

Drug Design, Development and Therapy is an international, peerreviewed open-access journal that spans the spectrum of drug design and development through to clinical applications. Clinical outcomes, patient safety, and programs for the development and effective, safe, and sustained use of medicines are the features of the journal, which

\section{Dovepress}

has also been accepted for indexing on PubMed Central. The manuscript management system is completely online and includes a very quick and fair peer-review system, which is all easy to use. Visit http://www.dovepress.com/testimonials.php to read real quotes from published authors. 